\title{
Intensité du travail et mobilité professionnelle
}

Work Intensity and Occupational Mobility

\section{Thomas Amossé et Michel Gollac}

\section{CpenEdition}

Journals

Édition électronique

URL : http://journals.openedition.org/travailemploi/2307

DOI : 10.4000/travailemploi.2307

ISSN : 1775-416X

\section{Éditeur}

DARES - Ministère du Travail

\section{Édition imprimée}

Date de publication : 17 mars 2008

Pagination : $59-73$

ISSN : 0224-4365

\section{Référence électronique}

Thomas Amossé et Michel Gollac, «Intensité du travail et mobilité professionnelle », Travail et Emploi [En ligne], 113 | janvier-avril 2008, mis en ligne le 17 février 2011, consulté le 01 mai 2019. URL : http:// journals.openedition.org/travailemploi/2307 ; DOI : 10.4000/travailemploi.2307 


\title{
Intensité du travail et mobilité professionnelle
}

\author{
Thomas Amossé(*), Michel Gollac(**)
}

\begin{abstract}
L'intensification du travail est-elle liée statistiquement à une augmentation de la mobilité des salariés? Cette hypothèse est confirmée ici empiriquement, à partir de l'enquête sur la Formation et la qualification professionnelle (FQP) de 2003. Mais le lien entre intensité et mobilité est-il de même nature pour tous? Une forte intensité du travail est associée à une probabilité accrue de mobilité descendante aussi bien que de mobilité ascendante. En début de carrière, l'intensité du travail favorise les mobilités horizontales ou ascendantes; mais la part des mobilités descendantes devient notable en milieu de carrière; enfin un travail trop intense pousse les plus âgés vers l'inactivité. Il n'est donc pas assuré qu'une forte intensité du travail soit compatible avec la construction de parcours professionnels satisfaisants pendant toute la durée de la vie active. Le maintien d'une telle intensité pourrait poser problème dans une société vieillissante. Même si les trajectoires ascendantes sont plus fréquentes que les trajectoires descendantes, les mobilités descendantes sont, subjectivement, plus marquantes que les mobilités ascendantes, de sorte qu'une intensité trop forte pourrait avoir, au total, un effet négatif sur le bien-être et la motivation des salariés.
\end{abstract}

Le travail intense, dont la diffusion a été, en France, une caractéristique marquante des années 1980 et 1990, est parfois opposé au travail soutenable, celui qui est compatible avec le maintien de la santé, des compétences et de l'employabilité (Docherty, Forslin, Shani, 2002). L'intensification du travail serait associée à une part de l'accroissement observé de la mobilité des salariés, et en particulier de la mobilité descendante. Cette intensification créerait ainsi une forme de «précarité du travail», pour reprendre l'expression de Serge Paugam (PAUGAM, 2000).

Résultat de l'analyse sociologique des récits de vie et situations de travail, cette thèse n'a pas encore fait l'objet d'un examen empirique sur grands échantillons. Elle mérite notamment d'être examinée plus directement à partir de données qui permettraient de relier les caractéristiques du travail et la trajectoire professionnelle des salariés. Peut-on mettre en évidence un lien statistique entre l'intensité du travail et la mobilité professionnelle? Ce lien est-il le même pour tous, résiste-t-il à la prise en compte des caractéristiques des individus, de leur poste et de leur entreprise? L'accroissement éventuel de la mobilité correspond-il à des évolutions positives

(*) Centre d'études de l'emploi, CEE; thomas.amosse@mail. enpc.fr

(**) Centre de recherche en économie et statistique, CREST

- Centre Maurice Halbwachs, CMH; michel.gollac@ensae.fr. ou négatives pour le salarié, du point de vue de son salaire ou de sa position socioprofessionnelle? Quelles sont les formes d'intensité du travail qui sont associées à davantage de mobilité? S'agit-il alors de fuir le travail intense?

Ce sont autant de questions auxquelles nous tenterons d'apporter des éléments de réponse et qui nous semblent à même d'éclairer les réformes en cours qui concernent le monde du travail. En effet, l'hypothèse d'un lien entre contenu du travail et trajectoire professionnelle rejoint l'exigence de prise en compte de la pénibilité du travail dans les réformes des retraites. Elle interroge aussi les compromis que traduisent les projets de flexicurité à la française: si les conséquences de l'intensité comme de la pénibilité ne se mesurent pas que du point de vue du travail et à court terme, mais aussi du point de vue des carrières et à plus long terme, pourquoi ne pas en tenir compte dans la sécurisation des parcours professionnels? En d'autres termes, si le travail intense créé des trajectoires professionnelles insatisfaisantes, ne serait-ce que pour certains travailleurs, ne faut-il pas tenter de le limiter et d'y apporter de justes contreparties?

Après avoir présenté la construction de notre hypothèse, nous précisons la démarche empirique que nous avons adoptée. La présentation détaillée des résultats fournira ensuite l'occasion d'une discussion sur leurs limites ainsi que leur portée, que nous résumerons en conclusion. 


\section{Problématique et formulation d'hypothèse}

Bien que l'intensité du travail soit une notion difficile à définir, et plus encore à mesurer, il n'y a guère de doute sur le fait que le travail s'est intensifié au cours des deux dernières décennies du vingtième siècle. Or de nombreuses études attestent de liens entre forte intensité du travail, mauvaises conditions de travail et problèmes de santé et permettent d'en comprendre les raisons. Ces travaux suggèrent que les effets de l'intensité sont différenciés: ses liens avec la mobilité pourraient donc l'être également.

\section{Formes et mesures de l'intensité du travail}

Nombreux sont les acteurs qui parlent de l'intensité du travail ou de ses synonymes: cadence, rythme, effort, etc. La notion ne se laisse pourtant pas aisément définir. En fait, il y a autant de formes d'intensité du travail qu'il y a de formes d'organisation (VALEYRE, 2004). Nous nous contenterons ici d'esquisser une typologie, qui suffit à donner une idée de la complexité du phénomène.

Une première forme d'intensité correspond à un travail homogène ou décomposable en un ensemble d'opérations homogènes: l'intensité désigne ici le nombre d'opérations par unité de temps. Elle est facilement mesurable et peut même être un objectif affiché pour le salarié, comme dans les systèmes de production dits «taylorien». À l'inverse, dans les formes modernes d'organisation, la direction renonce de plus en plus souvent à décomposer ainsi le travail, qui devient une «boîte noire». Seule est pilotée la relation entre les ressources (effectifs, matériels, etc. ) et les objectifs. Dans ce cadre, l'intensité correspond au travail qu'il faut au salarié pour atteindre les objectifs avec les ressources dont il dispose. Par ailleurs, de nombreuses organisations poursuivent des buts multiples : par exemple assurer la régularité qualitative et quantitative de la production et en même temps répondre aux fluctuations qualitatives et quantitatives de la demande; si tout ou partie de la conciliation de ces objectifs revient aux salariés, il en résulte une forme d'intensité qui a à voir avec la complexité du travail. Le changement organisationnel lui-même, par l'effort d'apprentissage et d'ajustement qu'il nécessite, est une source d'intensité du travail. Et un haut niveau d'intensité du travail tend à se perpétuer par un cercle vicieux de l'urgence et de l'impréparation: en mobilisant tout l'effort pour la tâche du moment, l'intensité du travail empêche de se préparer convenablement à la tâche future qui devra être exécutée avec moins de ressources, donc avec une plus grande intensité. Enfin, en dehors des contraintes venues de l'organisation, un travailleur peut de lui-même choisir de faire un effort élevé, par intérêt économique ou par intérêt désintéressé pour son travail.
La complexité de la notion d'intensité ne permet pas d'espérer disposer d'une mesure simple, précise ou complète de celle-ci, sauf peut-être en se limitant à analyser un très petit nombre de situations de travail. Deux types de mesure sont principalement utilisés dans la littérature. Les unes portent sur l'intensité subjectivement vécue par les travailleurs. Par exemple, dans l'enquête française sur les conditions de travail de 2005, on demande: «Êtes-vous obligé de vous dépêcher?». Dans l'enquête européenne, toujours sur les conditions de travail et également de 2005, on demande: "Votre rythme de travail vous impose-t-il de travailler à un rythme très élevé?». Une telle façon de faire a l'avantage d'être synthétique: peu importent les sources de l'intensité, on en mesure directement la résultante. Mais elle n'est pas sans inconvénient. Notamment, de telles appréciations peuvent être influencées par le degré général de satisfaction au travail, qui dépend en partie de facteurs sans lien direct avec son intensité.

Aussi utilise-t-on également des mesures plus objectives, le plus souvent en complément du questionnement subjectif. Ces mêmes enquêtes française et européenne sur les conditions de travail comportent par exemple une question qui entend préciser la variété des contraintes qui pèsent sur les rythmes de travail: "Votre rythme de travail vous est-il imposé par la cadence d'une machine ou le déplacement automatique d'un produit? (oui / non), des demandes extérieures du public? (oui / non), etc.». Ce type de mesure dépend peu de l'appréciation globale que les travailleurs portent sur leur travail. Certes, il peut arriver qu'un travailleur déclare qu'il doit respecter, par exemple, des délais d'un jour au plus tandis qu'un de ses collègues, dans la même situation de travail, ne les ressentira pas comme impératifs. Mais ceci n'est guère gênant car, de fait, dans cet exemple, les délais s'imposent au premier et pas au second. Par contre, ce genre de mesure ne permet de saisir que certaines formes d'intensité. De plus, on connaît l'existence de ces contraintes, pas leur niveau.

\section{L'intensification du travail au cours des dernières décennies}

Les enquêtes sur les conditions de travail ont permis de mettre en évidence une intensification du travail (CARTRON, 2004). Celle-ci a été très inégale selon les pays, à la fois dans son ampleur et dans son calendrier. En France, le mouvement d'intensification a débuté au milieu des années 1980. Il a été rapide jusqu'à la fin de la décennie suivante et, même si l'on semble connaître actuellement une pause dans l'intensification, la tendance ne s'est pas inversée depuis (BUÉ et al., 2007).

La forme particulière d'intensité créée par les organisations hybrides a particulièrement progressé. En 1984, 23\% des salariés étaient soumis à des contraintes de rythme industrielles, résultant de 
dispositifs destinés à assurer la régularité de la production: cadence automatique des machines, normes à respecter en un jour ou moins, etc. À la même date, $18 \%$ étaient soumis à la nécessité de répondre immédiatement à la demande des clients. Ces deux types de contrainte tendaient à s'exclure: seulement $4 \%$ des salariés connaissaient les deux. En 1998, les deux types de contraintes ont très fortement progressé : $45 \%$ pour la contrainte industrielle, $54 \%$ pour la contrainte marchande. Mais surtout de nombreuses organisations combinent organisation industrielle et organisation marchande et reportent sur leurs salariés les contraintes qui en résultent: $27 \%$ d'entre eux subissent à la fois les deux types de contrainte.

\section{Les effets de l'intensité du travail}

De nombreux travaux empiriques ont mis en évidence des effets néfastes de l'intensité du travail, $\mathrm{du}$ moins quand elle est excessive, sur la santé physique et psychique des salariés.

Les travaux pionniers de KARASEK (1984) puis KARASEK et THEøRELL (1990), complétés ensuite par de nombreuses études menées par ces auteurs ou leurs disciples, ont mis en évidence une augmentation à terme de la survenue de maladies, en particulier mentales et cardio-vasculaires, lorsque les exigences du travail sont, selon la perception du travailleur, fortes, que sa «latitude décisionnelle» (job latitude, incluant l'autonomie procédurale, les possibilités de perfectionner ses compétences, etc. ) est faible et qu'il ne bénéficie que d'un faible soutien social (émotionnel et technique). Johannes Siegrist a élaboré une variante du modèle de Karasek: lorsque les exigences du travail, perçues par le travailleur, sont fortes et que la reconnaissance (économique et sociale) du travail est faible, les risques de maladie sont accrus (SIEGRIST, 1996). Ce modèle a également été validé empiriquement, y compris sur données de panel (Bosma et al., 1998; NiEDHAMMER et al., 2004).

D'autres travaux complètent ceux-ci en établissant un lien instantané entre intensité du travail et conditions de travail. À l'issue d'une revue de la littérature anglophone, WiCHERT (2002) conclut à un lien positif entre intensité et pénibilités du travail. L'utilisation de sources indépendantes et de meilleure qualité confirme le lien, toutes choses égales par ailleurs, entre contraintes de rythme d'une part, pénibilités physiques et psychiques, nuisances et risques d'autre part (GolLAC, 2005). Une forte intensité perçue du travail accroît aussi le risque d'exprimer une forte souffrance au travail (BAUDELOT et al., 2003).

La littérature ergonomique explique bien le lien entre intensité du travail et conditions de travail (Lepla, Cuny, 1984). Les formes de travail qui conviennent aux personnes, qui leur permettent de maintenir leur santé et d'accroître leur capital humain sont très différentes d'un individu à l'autre (Laville, 1998). De ce fait les formes d'organisation qui créent une forte intensité du travail et limitent en conséquence la gamme des modes opératoires, conviennent bien à certains travailleurs, mais très mal à d'autres. Les travailleurs à qui elles ne conviennent pas connaissent de mauvaises conditions de travail et sont menacés de troubles de santé. Cependant, en raison de ce mode d'action, on peut supposer que l'effet de l'intensité n'est pas de dégrader uniformément les conditions de travail, mais d'augmenter leur variabilité interindividuelle, même au sein d'une catégorie de travailleurs occupant des postes analogues (Gollac, VolKoff, 2001).

Et, de fait, ces mêmes travaux, ainsi que d'autres, mettent en évidence des effets positifs de l'intensité $\mathrm{du}$ travail. Lorsque les exigences du travail sont, toujours selon la perception du travailleur, fortes, mais que sa «latitude décisionnelle» ainsi que le soutien social dont il bénéficie sont également forts, il tend à connaître un «développement personnel» (Karasek, Theørell, 1990). Ce résultat est sans doute contestable, tant parce que les auteurs sousestiment l'impact de la position sociale que parce que leur conception du développement personnel n'est pas exempte d'ethnocentrisme de classe. Néanmoins, il est conforté par d'autres observations (BAUDELOT et al., 2003): une forte intensité perçue du travail va de pair, toutes choses égales par ailleurs, avec des gratifications symboliques.

Au total, conformément à ce que les travaux des ergonomes laissaient supposer, les effets de l'intensité du travail sont ambigus ou, plus exactement inégaux. En réponse à la question «Seriez-vous heureux qu'un de vos enfants s'engage dans la même activité que vous?», qui mesure bien le bien-être au travail, les travailleurs qui rapportent une forte intensité de travail ne répondent guère moins «oui» que les autres, toutes choses égales par ailleurs: les «Non, c'est trop dur» des uns équilibrent les «Oui, c'est un travail passionnant» des autres.

\section{Quels effets de l'intensité sur la mobilité?}

Il est donc raisonnable de supposer que les effets d'une forte intensité du travail sur la carrière future seront variables, en fonction des individus et des circonstances. On peut distinguer deux situations polaires.

Premier cas: le travailleur dispose des moyens de faire face aux contraintes auxquelles il est soumis. Ces moyens peuvent être organisationnels: latitude décisionnelle, soutien social par exemple. Ils peuvent être personnels: éducation, capital social personnel, profil psychologique, particularités physiologiques. Ces ressources permettent à l'individu de réussir à faire son travail et de se maintenir en bonne santé (ce qui en retour lui permet de continuer à faire face). Réussite dans le travail et également bonne 
santé sont des facteurs d'accroissement des chances de promotion.

Second cas: le travailleur ne dispose pas des moyens, organisationnels et personnels, de faire face aux contraintes auxquelles il est soumis. Il ne réussit pas à faire son travail, ou ne réussit pas à se maintenir en bonne santé (ce qui en retour ne lui permet qu'encore plus difficilement de faire face). Échec dans le travail et également mauvaise santé réduisent les chances de promotion et augmentent les risques d'éviction (perte d'emploi, déclassement).

De plus, au sein d'une même organisation, le contraste est fort entre les travailleurs qui ont des ressources personnelles leur permettant de faire face à l'intensité du travail et leurs collègues moins favorisés (on peut supposer que les ressources organisationnelles sont les mêmes pour tous, bien que ce ne soit pas toujours le cas). Les premiers ont alors un avantage comparatif sur les seconds, d'autant plus que les fortes intensités de travail se rencontrent souvent dans des organisations qui ont développé des procédures d'évaluation du travail (Gollac, VolkofF, 1996): même si ces procédures sont souvent loin de capter la réalité du travail et l'ensemble des performances, elles rendent plus visible l'écart entre ceux qui satisfont les critères d'évaluation et ceux qui n'y parviennent pas, que ces critères soient pertinents ou non. La divergence des trajectoires en est, peut-on penser, d'autant accentuée.

$\mathrm{Au}$ total, on peut penser qu'une forte intensité du travail va de pair avec à la fois plus de mobilité ascendante et plus de mobilité descendante.

\section{Source et méthodes}

Nous allons maintenant montrer qu'il existe en effet un lien statistique entre intensité du travail et mobilité. Si le constat de ce lien ne prouve pas en lui-même que l'intensité «cause» la mobilité, il est cependant cohérent avec les mécanismes explicatifs qui viennent d'être décrits. Des études statistiques longitudinales et études de terrain spécifiques doivent pouvoir permettre de confirmer ultérieurement la pertinence de ce cadre d'interprétation et la nature causale du lien observé.

\section{Source statistique}

Nous nous proposons d'examiner l'impact à moyen-long terme de l'intensité du travail sur l'emploi et la qualification en utilisant une source longitudinale : l'enquête sur la Formation et la qualification professionnelle (FQP) réalisée en 2003 par l'Insee (InsEe, 2005). Cette enquête, réalisée en faceà-face au domicile des personnes interrogées, porte sur un échantillon de 39300 personnes. Elle propose une mesure objective de l'intensité (contraintes de rythme) à cinq ans d'intervalle et une description des carrières durant ces cinq années.

Dans cette enquête, les salariés indiquent les contraintes de rythme qui pèsent sur leur emploi au moment de l'enquête, ainsi que cinq ans auparavant à l'aide de questions rétrospectives. Les questions posées sont:

Votre rythme de travail est-il imposé par:

- la cadence automatique d'une machine ou le déplacement d'un produit? (oui / non);

- des normes de production ou des délais à respecter en un jour au plus? (oui / non);

- une demande extérieure du public? (oui / non);

- les contrôles ou surveillance au moins quotidiens de la hiérarchie? (oui / non).

On notera que la référence à « une demande extérieure du public» est réductrice et risque de faire omettre la dépendance à la demande d'autres entreprises, par exemple. Par ailleurs, la mesure rétrospective des contraintes de rythme, comme d'ailleurs des conditions de travail en général, ne va pas sans poser problème (MoliniÉ, 2003). Cette question sera examinée plus en détail ci-dessous.

Outre une description fine des situations d'emploi à ces deux dates, on dispose également dans l'enquête d'un calendrier recensant l'ensemble des postes occupés pendant les cinq ans, c'est-à-dire sur la période 1998-2003. Pour chaque changement de poste, on sait en particulier si le salaire a augmenté, diminué ou est resté stable.

À partir de l'enquête FQP, on peut ainsi caractériser la mobilité professionnelle à cinq ans à la fois du point de vue de la qualification de l'emploi, de l'évolution des salaires ou du passage par le chômage ou l'inactivité, et du point de vue de l'intensité du travail. Il est donc possible d'examiner dans quelle mesure les parcours professionnels sont liés à l'intensité du travail et, en miroir, de comprendre comment l'intensité du travail évolue au gré des parcours: est-ce que les travailleurs soumis à une très forte intensité du travail sont plus sujets à des baisses de salaire? Sont-elles une contrepartie à l'obtention d'un travail moins exigeant? En d'autres termes, les déclassements professionnels sont-ils liés à l'impossibilité de soutenir des contraintes de rythme élevées? Ou à l'inverse, l'intensité du travail est-elle un moyen d'accéder plus facilement à des promotions? En ce cas, l'intensité du travail reste-t-elle élevée?

\section{Indicateurs et modélisations statistiques}

Le champ de notre étude est constitué des individus ayant un emploi salarié cinq ans avant la date de l'enquête, c'est-à-dire en 1998. Ils sont au nombre de 22800 dans l'échantillon.

Comme indicateur d'intensité, nous utilisons le nombre de réponses positives aux questions sur les contraintes de rythme en 1998 . Un bon quart des 
salariés n'étaient, selon leurs déclarations, soumis en 1998 à aucune contrainte de rythme. Près du tiers étaient soumis à une seule contrainte, un quart à deux contraintes et un sixième à trois ou plus ( $c f$. tableau 1).

Tableau 1

Cumul de contraintes de rythme en 1998

\begin{tabular}{|c|c|}
\hline $\begin{array}{c}\text { Nombre de contraintes } \\
\text { déclarées }\end{array}$ & Part de salariés \\
\hline 0 & $26,2 \%$ \\
\hline 1 & $30,1 \%$ \\
\hline 2 & $25,3 \%$ \\
\hline 3 ou 4 & $18,4 \%$ \\
\hline Total & $100 \%$ \\
\hline
\end{tabular}

Lire ainsi: $26,2 \%$ des salariés de 15 à 59 ans en 1998 ont déclaré qu'en 1998, ils n'étaient alors soumis à aucune des contraintes de rythme envisagées par le questionnaire.

Champ: salariés de 15 à 59 ans en 1998; déclarations rétrospectives.

Source: enquête FQP (2003).

Nous étudions divers aspects de la mobilité. Par mobilité, nous entendons ici changement de poste, qu'il s'accompagne ou non d'un changement d'entreprise. Tout d'abord, nous mesurons le nombre de transitions au cours de la période 1998-2003. Nous pouvons aussi classer les trajectoires en ascendantes, stables ou descendantes. Pour cela, deux méthodes peuvent être utilisées. La première consiste à comparer la catégorie socioprofessionnelle en 1998 et celle en 2003. Sont considérées comme ascendantes les trajectoires qui font passer de profession intermédiaire vers cadre, d'ouvrier ou employé vers profession intermédiaire ou cadre, et d'ouvrier ou employé non qualifié(1) vers ouvrier ou employé qualifié. Les trajectoires inverses sont considérées comme descendantes, les autres sont réputées horizontales. La deuxième méthode consiste à créer un indicateur de mobilité salariale. La mobilité salariale sera dite ascendante si le nombre de transitions s'accompagnant d'une hausse de salaire est plus élevé que le nombre de transitions s'accompagnant d'une baisse; descendante si c'est l'inverse; horizontale si les deux s'équilibrent. Enfin, nous étudions aussi la première mobilité: la date de sa survenue et la forme qu'elle a prise.

Nous utilisons ces mesures de l'intensité et de la mobilité pour estimer le lien entre elles «toutes choses (mesurées) égales par ailleurs ». Nous traitons la mobilité comme variable expliquée et l'intensité comme variable explicative. Ceci est conforme à la causalité que nous souhaitons explorer. Nous ne prétendons pas pour autant mesurer des liens «purement» causaux: les problèmes que pose une telle mesure sont discutés ci-dessous.

Une première modélisation statistique consiste à « expliquer» l'intensité de la mobilité, autrement dit le nombre de mobilités. Ce nombre restant faible et la forme de la fonction lien l'intensité à la mobilité étant inconnue, il nous a paru préférable de le traiter comme une variable discrète. L'estimation se fait à l'aide d'un logit ou d'un probit multinomial ordonné.

Un second type de modélisation consiste à «expliquer» la survenue d'un événement. Il peut s'agir de la survenue d'une mobilité: on utilise alors un probit ou un logit binomial. Il peut s'agir de différents types d'états (CS par exemple) possibles en 2003 compte tenu de l'état en 1998: la méthode d'estimation est dans ce cas un probit ou logit multinomial (non ordonné).

Enfin, un troisième type de modélisation porte sur la date de survenue de la première mobilité. Nous utilisons ici un modèle de durée semi-paramétrique à durée de vie accélérée. Il serait possible de séparer les différents types de mobilité, grâce à un modèle de durée à risques concurrents, mais nous n'avons pas procédé à ce travail. En effet, les modèles de durée posent de toute façon des problèmes méthodologiques délicats en raison de la «censure à gauche» des données, c'est-à-dire du fait que l'on n'observe que les cinq dernières années des périodes d'emploi ayant débuté avant 1998 .

\section{Résultats empiriques}

Les résultats obtenus sont compatibles avec notre hypothèse de départ et ce quelle que soit la méthode statistique employée.

\section{L'intensité accroît la mobilité}

«Toutes choses inégales réunies», une plus forte intensité en 1998 est associée à un nombre plus élevé de mobilités entre 1998 et 2003 ( $c f$. tableau 2). Parmi les salariés de 1998 qui déclarent qu'à cette date, ils n'étaient soumis à aucune contrainte de rythme, la moitié $(49,4 \%)$ n'a pas changé de poste au cours des cinq années suivantes. C'est le cas de seulement 40,2\% de ceux qui cumulaient au moins trois contraintes de rythme. Inversement, 7,0\% de ces derniers ont changé au moins trois fois de poste, ce qui n'est le cas que de 3,8\% des salariés abrités des contraintes en 1998. 
Tableau 2

Changements de postes entre 1998 et 2003 en fonction du cumul de contraintes de rythme en 1998

\begin{tabular}{|c|c|c|c|c|c|}
\hline \multirow{2}{*}{$\begin{array}{c}\text { Nombre de } \\
\text { contraintes } \\
\text { déclarées }\end{array}$} & \multicolumn{5}{|c|}{ Nombre de mobilités } \\
\hline & 0 & 1 & 2 & $\begin{array}{l}3 \text { et } \\
\text { plus }\end{array}$ & Ensemble \\
\hline 0 & $49,4 \%$ & $41,0 \%$ & $5,2 \%$ & $3,8 \%$ & $100 \%$ \\
\hline 1 & $46,4 \%$ & $41,2 \%$ & $7,8 \%$ & $5,1 \%$ & $100 \%$ \\
\hline 2 & $41,6 \%$ & $44,8 \%$ & $7,8 \%$ & $5,8 \%$ & $100 \%$ \\
\hline 3 ou 4 & $40,2 \%$ & $44,8 \%$ & $7,8 \%$ & $7,0 \%$ & $100 \%$ \\
\hline
\end{tabular}

Lire ainsi : 49,4\% des salariés qui n'ont déclaré aucune contrainte de rythme en 1998 n'ont connu aucune mobilité entre 1998 et 2003.

Champ: salariés de 15 à 59 ans en 1998; déclarations rétrospectives.

Source: enquête FQP (2003).

Une analyse «toutes choses égales par ailleurs» à l'aide d'un logit multinomial ordonné confirme ces résultats ( $c f$. tableau 3 ). Si on contrôle le secteur d'activité, la taille de l'entreprise, la catégorie socioprofessionnelle, le fait d'être à temps complet ou partiel, l'ancienneté dans le poste et la trajectoire salariale à l'entrée dans le poste (toutes informations relatives à la situation en 1998), ainsi que le sexe, l'âge, le diplôme et la nationalité, on trouve que les probabilités de connaître une mobilité et a fortiori deux, trois ou plus, sont significativement (au sens statistique et au sens sociologique) plus élevées lorsque l'individu était en 1998 soumis à une contrainte de rythme, par rapport à la situation où il n'en subit aucune; et qu'elles sont encore plus élevées lorsqu'il y a cumul d'au moins deux contraintes de rythme. Par contre, qu'il y ait trois ou quatre contraintes n'augmente pas significativement la mobilité par rapport au cas où il n'y en a que deux.

Tableau 3

Changements de postes entre 1998 et 2003 en fonction du cumul de contraintes de rythme en 1998 (résultats d'un logit multinomial ordonné)

\begin{tabular}{|l|c|c|c|}
\hline $\begin{array}{l}\text { Nombre de } \\
\text { contraintes } \\
\text { déclarées }\end{array}$ & Coefficient & $\begin{array}{c}\text { Écart type } \\
\text { du coefficient }\end{array}$ & $\begin{array}{c}\text { Rapport des } \\
\text { rapports de } \\
\text { chances }\end{array}$ \\
\hline $\begin{array}{l}0 \\
\text { (référence) }\end{array}$ & 0 & $/ / /$ & 1 \\
\hline 1 & $+0,11 * * *$ & 0,036 & $1,12 * * *$ \\
\hline 2 & $+0,29 * * *$ & 0,037 & $1,34 * * *$ \\
\hline 3 ou 4 & $+0,30 * * *$ & 0,042 & $1,35 * * *$ \\
\hline
\end{tabular}

Variables contrôlées: secteur d'activité en 1998, taille de l'entreprise, catégorie socioprofessionnelle, fait d'être à temps complet ou partiel, ancienneté et trajectoire salariale à l'entrée dans le poste à cette même date, ainsi que le sexe, l'âge et la nationalité

***: significativement différent de 0 ou de 1 au seuil $1 \%$.

Lire ainsi : à variables contrôlées égales, la probabilité d'avoir changé de postes entre 1998 et 2003 est plus élevée pour les salariés ayant déclaré avoir été exposés à une contrainte de rythme en 1998 (sur les quatre figurant dans le questionnaire) que pour ceux n'ayant déclaré aucune contrainte de rythme. Le rapport des rapports de chances est de 1,12: il est significativement différent de 1 au seuil $1 \%$.

Champ: salariés de 15 à 59 ans en 1998; déclarations rétrospectives. Source: enquête FQP (2003).
Nous avons également utilisé un modèle de durée semi-paramétrique à durée de vie accélérée (tableau non reproduit ici, $c f$. Amossé, Gollac, 2007; $c f$. tableau 4). Les variables de contrôle sont les mêmes que dans le logit multinomial décrit à l'alinéa précédent et les résultats sont tout à fait concordants. Toutes choses égales par ailleurs, la durée dans le même poste est significativement plus faible quand ce poste comporte une contrainte de rythme par rapport au cas où il n'en implique pas. Il est encore significativement plus faible si deux contraintes (ou éventuellement plus) se cumulent. Les données étant censurées à gauche, nous avons testé la robustesse de ce résultat en séparant les individus en trois classes selon leur ancienneté dans le poste en 1998: moins d'un an, un à cinq ans, plus de cinq ans (tableau non reproduit ici, $c f$. Amossé, Gollac, 2007; $c f$. tableau 5). L'effet du cumul de contraintes de rythme apparaît un peu moindre pour les salariés nouvellement embauchés. Il reste toutefois statistiquement significatif même dans leur cas.

\section{L'intensité du travail fait diverger les carrières}

On peut s'attendre à ce qu'une forte intensité crée une forte divergence entre ceux qui réussissent à $\mathrm{y}$ faire face et leurs collègues moins chanceux. C'est ce que confirment nos résultats. Le cumul de contraintes de rythme rend plus probables tous les types de mobilité, mais plus particulièrement ceux qui se traduisent par une promotion ou une régression économique ou sociale.

L'examen des simples statistiques «toutes choses inégales réunies» montre que les trajectoires de mobilité salariale ascendantes comme descendantes, entre 1998 et 2003 sont d'autant plus fréquentes que le salarié cumule les contraintes de rythme en début de période ( $c f$. tableau 4). Par trajectoire ascendante nous entendons ici une trajectoire où les changements de poste avec hausse de salaire sont plus nombreux que ceux avec baisse et vice versa pour les trajectoires descendantes. Les cas de mobilité ascendante représentent $17,8 \%$ des salariés abrités des contraintes de rythme en 1998 , mais $24,0 \%$ de ceux cumulant au moins trois contraintes. La différence peut paraître plus faible dans le cas de carrières descendantes: $7,4 \%$ contre $5,1 \%$. Mais il faut voir que l'écart porte sur des cas relativement rares, l'ascension salariale étant toujours le cas le plus fréquent. En réalité le rapport des rapports de chances est à peu près le même, voire un peu supérieur. Les trajectoires de mobilité où les épisodes de baisse et de hausse du salaire s'équilibrent sont aussi plus fréquentes pour les salariés soumis en 1998 à une forte intensité de travail, mais l'écart avec ceux abrités est modéré. L'intensité paraît donc, «toutes choses inégales réunies», augmenter toutes les formes de mobilité (sauf les sorties de l'emploi 
Tableau 4

Mobilité salariale entre 1998 et 2003 en fonction du cumul de contraintes de rythme en 1998

\begin{tabular}{|c|c|c|c|c|c|c|}
\hline \multirow{2}{*}{$\begin{array}{c}\text { Nombre de } \\
\text { contraintes } \\
\text { déclarées }\end{array}$} & \multirow{2}{*}{ Pas de mobilité } & \multicolumn{3}{|c|}{ Évolution du salaire } & \multirow{2}{*}{$\begin{array}{l}\text { Mobilité sans } \\
\text { retour en } \\
\text { emploi }\end{array}$} & \multirow{2}{*}{ Ensemble } \\
\hline & & Augmentation & Stabilité & Diminution & & \\
\hline 0 & $49,4 \%$ & $17,8 \%$ & $15,1 \%$ & $5,1 \%$ & $12,7 \%$ & $100 \%$ \\
\hline 1 & $46,4 \%$ & $20,4 \%$ & $15,6 \%$ & $5,7 \%$ & $11,9 \%$ & $100 \%$ \\
\hline 2 & $41,6 \%$ & $23,3 \%$ & $16,5 \%$ & $6,6 \%$ & $12,0 \%$ & $100 \%$ \\
\hline 3 ou 4 & $40,2 \%$ & $24,0 \%$ & $17,8 \%$ & $7,4 \%$ & $10,6 \%$ & $100 \%$ \\
\hline
\end{tabular}

Augmentations du salaire: trajectoire où les changements de poste avec hausse de salaire sont plus nombreux que ceux avec baisse et vice versa pour les «diminutions du salaire».

Lire ainsi : 17,8\% des salariés qui n'ont déclaré aucune contrainte de rythme en 1998 ont connu une trajectoire avec plus de hausses que de baisses de salaire entre 1998 et 2003 .

Champ: salariés de 15 à 59 ans en 1998; déclarations rétrospectives.

Source: enquête FQP (2003).

Tableau 5

Mobilité socioprofessionnelle des ouvriers et employés qualifiés et des professions intermédiaires, entre 1998 et 2003, en fonction du cumul de contraintes de rythme en 1998

\begin{tabular}{|l|c|c|c|c|c|c|}
\hline \multirow{2}{*}{$\begin{array}{c}\text { Nombre de } \\
\text { contraintes } \\
\text { déclarées }\end{array}$} & \multirow{2}{*}{ Pas de mobilité } & \multicolumn{2}{|c|}{ Évolution socioprofessionnelle } & $\begin{array}{c}\text { Mobilité sans } \\
\text { retour en }\end{array}$ & \multirow{2}{*}{ Ensemble } \\
\cline { 3 - 6 } & & Promotion & Stabilité & Déclassement salarié & \\
\hline 0 & $50,2 \%$ & $6,4 \%$ & $31,1 \%$ & $4,0 \%$ & $8,4 \%$ & $100 \%$ \\
\hline 1 & $48,2 \%$ & $7,4 \%$ & $31,5 \%$ & $4,9 \%$ & $8,1 \%$ & $100 \%$ \\
\hline 2 & $43,2 \%$ & $7,5 \%$ & $35,9 \%$ & $4,9 \%$ & $8,4 \%$ & $100 \%$ \\
\hline 3 ou 4 & $42,3 \%$ & $9,0 \%$ & $35,6 \%$ & $5,7 \%$ & $7,4 \%$ & $100 \%$ \\
\hline
\end{tabular}

Promotion: passage d'ouvrier ou employé qualifié à profession intermédiaire ou cadre ou de profession intermédiaire à cadre; déclassement: mouvements inverses; stabilité: autres cas.

Lire ainsi : 6,4\% des employés et ouvriers qualifiés et professions intermédiaires qui n’ont déclaré aucune contrainte de rythme en 1998 ont connu une promotion entre 1998 et 2003.

Champ: salariés de 15 à 59 ans en 1998 appartenant aux catégories d'ouvriers qualifiés, d'employés qualifiés ou de professions intermédiaires ; déclarations rétrospectives.

Source: enquête FQP (2003).

salarié (2), résultat qui est paradoxal et s'explique, comme on va le voir dans un instant, par des effets de structure), mais surtout augmenter la mobilité ascendante et la mobilité descendante.

On peut évidemment critiquer notre définition des carrières ascendantes et descendantes à partir du nombre de transitions associées à une hausse ou une baisse de salaire: l'ampleur de ces hausses et baisses n'est pas connue et une forte baisse peut, par exemple, plus qu'annuler l'effet de deux hausses (3). Pour cette raison, nous avons examiné de façon complémentaire la mobilité en termes de catégorie socioprofessionnelle. Cette façon de faire a aussi

(2) Ceci accumule des trajectoires très hétérogènes, conduisant à l'emploi non salarié, à l'inactivité ou au chômage. Nous étudierons plus particulièrement le risque de chômage dans un approfondissement de ce travail.

(3) Même si, les cas de mobilités multiples étant rares, les trajectoires comportant plusieurs hausses et baisses de salaire le sont encore davantage (moins d'un pour-cent de l'échantillon total). ses inconvénients. D'une part, la mesure de la catégorie socioprofessionnelle est sujette à un flou de déclaration et à un flou de chiffrement (DESRosières, THÉVEnOT, 1988). D'autre part, les petites progressions ou régressions peuvent n'être pas enregistrées. Et on ne peut pas du tout enregistrer les reculs subis par des individus déjà non qualifiés au départ (mais pas forcément au plus bas de l'échelle des coefficients), non plus que les progressions de ceux qui, en 1998, avaient déjà le statut de cadre. Ceci oblige à exclure du champ (pour cette analyse seulement) tant les salariés non qualifiés que les cadres. Ceci doit amener à une prudence particulière dans l'interprétation des résultats (4). Ces préventions étant posées, nous observons en ce qui concerne les chan-

(4) On ne peut a priori pas exclure l'idée que les liens entre mobilité et intensité sont différents pour ces catégories en haut et en bas de la hiérarchie des emplois de ce qu'ils sont aux autres niveaux de qualification: en particulier, une grande partie des cadres comme des travailleurs non qualifiés ont des activités de service, qui sont particulièrement difficiles à évaluer. 
Tableau 6

Mobilité salariale entre 1998 et 2003 en fonction du cumul de contraintes de rythme en 1998 (résultats d'un logit multinomial, estimation des coefficients)

\begin{tabular}{|l|c|c|c|c|c|}
\hline $\begin{array}{c}\text { Nombre de } \\
\text { contraintes décla- } \\
\text { rées }\end{array}$ & $\begin{array}{c}\text { Pas de mobilité } \\
\text { (référence) }\end{array}$ & \multicolumn{3}{|c|}{ Évolution du salaire } & \multirow{2}{*}{$\begin{array}{c}\text { Mobilité sans } \\
\text { retour en emploi }\end{array}$} \\
\hline & & Augmentation & Stabilité & Diminution & \\
\hline 0 (référence) & 0 & 0 & 0 & 0 & 0 \\
\hline 1 & 0 & $+0,15 * * *$ & $+0,05$ & $+0,13$ & $+0,05$ \\
\hline 2 & 0 & $+0,39 * *$ & $+0,25 * * *$ & $+0,38^{* * *}$ & $+0,18^{* * *}$ \\
\hline 3 ou 4 & 0 & $+0,40 * * *$ & $+0,31 * * *$ & $+0,50 * * *$ & $+0,14 *$ \\
\hline
\end{tabular}

Augmentations du salaire: trajectoire où les changements de poste avec hausse de salaire sont plus nombreux que ceux avec baisse et vice versa pour les «diminutions du salaire».

Variables contrôlées: secteur d'activité en 1998, taille de l'entreprise, catégorie socioprofessionnelle, fait d'être à temps complet ou partiel, ancienneté dans le poste et trajectoire salariale à l'entrée dans le poste à cette même date, ainsi que le sexe, l'âge et la nationalité.

$*$ : significativement différent de 0 au seuil $10 \%, * *$ : significativement différent de 0 au seuil $5 \%$, ***: significativement différent de 0 au seuil $1 \%$.

Lire ainsi: à variables contrôlées égales, l'écart entre la probabilité d'avoir connu plus de hausses que de baisses de salaire entre 1998 et 2003 et celle de n'avoir pas connu de mobilité est plus élevé pour les salariés ayant déclaré avoir été exposés à une contrainte de rythme (sur les quatre figurant dans le questionnaire) en 1998 que pour ceux n'ayant déclaré aucune contrainte de rythme (l'écart est significatif au seuil $1 \%$ ).

Champ: salariés de 15 à 59 ans en 1998; déclarations rétrospectives.

Source: enquête FQP (2003).

Tableau 7

Changements de postes entre 1998 et 2003 en fonction des différentes contraintes de rythme en 1998 (résultats d'un logit multinomial ordonné)

\begin{tabular}{|l|c|c|c|}
\hline \multicolumn{1}{|c|}{ Contraintes déclarées } & Coefficient & Écart type du coefficient & $\begin{array}{c}\text { Rapport des rapports de } \\
\text { chances }\end{array}$ \\
\hline Cadence automatique & $+0,043$ & 0,053 & 1,04 \\
\hline Norme ou délai & $+0,094 * * *$ & 0,031 & $1,10 * * *$ \\
\hline Demande & $-0,047 *$ & 0,028 & $0,95 *$ \\
\hline Contrainte hiérarchique & $+0,280 * * *$ & 0,029 & $1,32 * * *$ \\
\hline
\end{tabular}

Variables contrôlées: secteur d'activité en 1998, taille de l'entreprise, catégorie socioprofessionnelle, fait d'être à temps complet ou partiel, ancienneté dans le poste et trajectoire salariale à l'entrée dans le poste à cette même date, ainsi que le sexe, l'âge et la nationalité.

***: significativement différent de 0 ou de 1 au seuil $1 \%$, *: significativement différent de 0 ou de 1 au seuil $10 \%$.

Lire ainsi: à variables contrôlées égales, la probabilité d'avoir changé de postes entre 1998 et 2003 est plus élevée pour les salariés ayant déclaré avoir été exposés à une contrainte de rythme liée à la cadence automatique d'une machine en 1998 que pour ceux n'ayant pas déclaré cette contrainte de rythme. Toutefois, le rapport des rapports de chances estimé est de 1,04: il n'est pas significativement différent de 1 au seuil $10 \%$. Champ: salariés de 15 à 59 ans en 1998; déclarations rétrospectives. Source: enquête FQP (2003).

gements de qualification des résultats parfaitement convergents avec ceux relatifs à la mobilité salariale. «Toutes choses inégales réunies », l'intensité augmente toutes les formes de mobilité socioprofessionnelle (sauf les sorties de l'emploi salarié), mais surtout elle augmente la mobilité ascendante et la mobilité descendante ( $c f$. tableau 5).

Les analyses «toutes choses égales par ailleurs», à l'aide de logits multinomiaux (non ordonnés) confirment et même renforcent cette conclusion, qu'il s'agisse de mobilité salariale ou de mobilité socioprofessionnelle. Dans les deux cas, nous contrôlons le secteur d'activité, la taille de l'entreprise, la catégorie socioprofessionnelle, le fait d'être à temps complet ou partiel, l'ancienneté dans le poste et la trajectoire salariale à l'entrée dans le poste (toujours en 1998), ainsi que le sexe, l'âge, le diplôme et la nationalité.

En ce qui concerne l'évolution des salaires, les trajectoires ascendantes et les trajectoires descendantes sont plus probables pour les salariés soumis à une contrainte de rythme et, plus encore, pour ceux qui subissent un cumul de contraintes ( $c f$. tableau 6). Les trajectoires comportant une mobilité, mais où hausses et baisses de salaires sont aussi nombreuses sont également plus probables et il en est de même, mais moins nettement (seul le cumul de contraintes a un effet significatif, modéré), des mobilités aboutissant à une sortie de 1'emploi. Les résultats concernant la mobilité socioprofessionnelle sont, à quelques détails près, analogues à ceux concernant la mobilité salariale (tableau non reproduit ici, $c f$. Amossé, Gollac, 2007, $c f$. tableau 9). Ils confirment 
donc que l'intensité du travail induit une divergence des trajectoires professionnelles.

Une forte intensité du travail est donc associée à un accroissement de la mobilité en général, même si c'est particulièrement le cas de la mobilité ascendante et descendante. Il est fort possible qu'une partie seulement du supplément de mobilité ascendante et descendante soit attribuable aux mécanismes décrits dans la littérature ergonomique. Une autre partie pourrait provenir de pratiques de gestion des ressources humaines spécifiquement flexibles dans les organisations provoquant de fortes contraintes de travail: en ce sens, cela ne serait pas parce que les salariés peuvent, ou non, faire face à l'intensité du travail qu'ils quittent leur poste, mais parce que ces postes sont prévus comme tels dans l'organisation du travail. Il est par ailleurs également possible que des travailleurs cherchent à quitter les postes à forte intensité, sans que cela soit directement lié à cette intensité, du fait de caractéristiques non observées de ces postes.

\section{Des effets différenciés selon la nature des contraintes de rythme}

Comme on l'a vu supra, ce n'est pas tant la contrainte en elle-même qui pose problème que la disparité entre elle et les ressources disponibles pour $\mathrm{y}$ faire face. Or les différentes formes de contrainte temporelle sont associées à des contextes organisationnels et technologiques différents. Il n'y a pas de description de ce contexte organisationnel dans l'enquête FQP (dont ce n'est pas le propos), de sorte que le coefficient associé à chaque forme d'intensité capture en fait une part des effets de contexte. On peut donc s'attendre à ce que les formes d'intensité souvent associées à des contextes organisationnels fragilisants aient un impact, au moins apparent, plus élevé sur les carrières.

C'est de très loin, la pression sur le rythme de travail exercée par «les contrôles ou surveillance au moins quotidiens de la hiérarchie» qui sont le plus fortement associés à une mobilité ultérieure (cf. tableau 7). Bien que statistiquement significatif, l'effet apparent des normes de production ou des délais à respecter en un jour au plus est nettement moins élevé. Quant à celui de la cadence automatique d'une machine ou du déplacement d'un produit, il est du même ordre de grandeur, mais non significativement différent de zéro. Enfin, le coefficient estimé associé à la «demande extérieure du public » est même négatif (bien qu'on ne puisse tout à fait écarter l'idée que ceci soit dû à une simple particularité de l'échantillon interrogé).

Des normes de production ou des délais à respecter en un jour au plus signalent un environnement industriel ou bureaucratique et constituent par eux-mêmes une limitation de l'autonomie des travailleurs concernés. Or la «latitude décisionnelle» dont ceux-ci disposent a un impact sur leur état de santé (KARASEK, 1984). Leur effet pourrait donc être double: perte de latitude décisionnelle et accroissement des exigences du travail. Tel est peut-être moins le cas de la demande des clients. La formulation de la question dans l'enquête FQP ne précise en effet pas que cette réponse doit être satisfaite immédiatement, ni même dans des délais courts. La contrainte qu' elle engendre peut donc être de faible intensité et ne comporter aucune atteinte à l'autonomie procédurale.

Pourquoi est-ce la pression hiérarchique qui a le plus fort impact? Il subsiste, certes, bon nombre d'organisations traditionnelles où la hiérarchie dicte la cadence. Mais, dans les formes modernes d'organisation, le rôle de la hiérarchie est censé, en principe, décliner. La hiérarchie est supposée n'avoir plus qu'à contrôler le respect de contraintes dont l'origine est marchande, bureaucratique ou technologique. En pratique, il n'en est rien. Les grandes transformations organisationnelles des années 1980 et 1990 ont au contraire vu la hiérarchie intervenir pour déterminer le rythme de travail d'un nombre croissant de salariés. Les contrôles de la hiérarchie sont particulièrement fréquents dans les organisations hybrides, où les salariés cumulent déjà plusieurs autres contraintes de rythme ( $c f$. tableau 8). En effet, les organisations récentes et complexes fonctionnent rarement bien. L'intervention en personne des membres de leur hiérarchie est nécessaire pour pallier leurs nombreux dysfonctionnements. Ces dysfonctionnements sont une source d'intensité, ou du moins une épreuve, pour les salariés dont ils affectent le travail. Ce serait ainsi les organisations qui fonctionnent mal qui entraîneraient une plus forte mobilité de leurs membres.

Tableau 8

La contrainte hiérarchique, un lien avec le cumul d'autres contraintes

\begin{tabular}{|c|c|}
\hline $\begin{array}{c}\text { Nombre de contraintes non } \\
\text { hiérarchiques }\end{array}$ & $\begin{array}{c}\text { Part des salariés soumis à } \\
\text { la contrainte hiérarchique }\end{array}$ \\
\hline 0 & $13 \%$ \\
\hline 1 & $26 \%$ \\
\hline 2 & $39 \%$ \\
\hline 3 & $46 \%$ \\
\hline 4 & $52 \%$ \\
\hline
\end{tabular}

Lire ainsi: parmi les salariés ayant déclaré n'être soumis à aucune contrainte de rythme en dehors de la dépendance au contrôle permanent de leur chef, $13 \%$ voient leur rythme dépendre de ce contrôle. Champ: salariés.

Source: enquête Dares-Insee de 2005 sur les conditions de travail.

Une explication alternative peut évidemment être avancée: la hiérarchie interviendrait auprès des salariés qui ne parviennent pas à répondre aux prescriptions. Ce serait, en quelque sorte, la «mauvaise qualité» des salariés qui serait à l'origine de la contrainte hiérarchique et qui entraînerait l'éviction de certains d'entre eux. Toutefois cette interpréta- 
Tableau 9

Effet de la contrainte de rythme hiérarchique sur la mobilité salariale entre 1998 et 2003 (estimation par la méthode du score de propension)

\begin{tabular}{|l|c|c|c|c|c|}
\hline \multirow{2}{*}{ Nombre de contraintes déclarées } & \multirow{2}{*}{ Pas de mobilité } & \multicolumn{3}{|c|}{ Évolution du salaire } & $\begin{array}{c}\text { Mobilité sans } \\
\text { retour en } \\
\text { emploi }\end{array}$ \\
\cline { 3 - 6 } & & Augmentation & Stabilité & Diminution & $11,9 \%$ \\
\hline Moyenne & $44,9 \%$ & $21,1 \%$ & $16,1 \%$ & $6,1 \%$ & $+0,5 \%$ \\
\hline Effet (en points) & $-6,7 \% * * *$ & $+3,3 \% * * *$ & $+1,3 \% * * *$ & $+1,5 \% * * *$ & $+0,5 \%$ \\
\hline $\begin{array}{l}\text { Effet (en rapport de rapports de } \\
\text { chances) }\end{array}$ & réf & $1,59 * * *$ & $1,44 * * *$ & $1,67 * * *$ & $1,38 * * *$ \\
\hline
\end{tabular}

Augmentations du salaire: trajectoire où les changements de poste avec hausse de salaire sont plus nombreux que ceux avec baisse et vice versa pour les «diminutions du salaire».

Variables contrôlées: secteur d'activité en 1998, taille de l'entreprise, catégorie socioprofessionnelle, fait d'être à temps complet ou partiel et ancienneté dans le poste à cette même date, ainsi que le sexe, l'âge et la nationalité.

*: significativement différent de 0 au seuil $10 \%, * *$ : significativement différent de 0 au seuil $5 \%, * * *$ : significativement différent de 0 au seuil $1 \%$.

Lire ainsi : à variables contrôlées égales, la probabilité de ne pas avoir changé de postes entre 1998 et 2003 est diminuée de 6,7\% pour les salariés dont le rythme de travail dépend des contrôles au moins quotidiens de la hiérarchie Cet écart est significativement différent de 0 au seuil $1 \%$.

Champ: salariés de 15 à 59 ans en 1998; déclarations rétrospectives.

Source: enquête FQP (2003).

Tableau 10

Évolution de l'intensité et évolution salariale pour les salariés soumis aux quatre contraintes de rythme en 1998

\begin{tabular}{|l|c|c|c|c|c|c|}
\hline \multirow{2}{*}{$\begin{array}{c}\text { Évolution de l'intensité } \\
\text { entre 1998 et 2003 }\end{array}$} & \multirow{2}{*}{$\begin{array}{c}\text { Pas de } \\
\text { mobilité }\end{array}$} & \multicolumn{2}{|c|}{ Évolution du salaire } & $\begin{array}{c}\text { Mobilité sans } \\
\text { retour en } \\
\text { emploi }\end{array}$ & \multirow{2}{*}{ Ensemble } \\
\cline { 3 - 7 } & Augmentation & Stabilité & Diminution & \\
\hline Stabilité & $68,8 \%$ & $16,4 \%$ & $11,2 \%$ & $3,6 \%$ & $/$ & $100 \%$ \\
\hline Diminution & $26,5 \%$ & $36,5 \%$ & $23,8 \%$ & $13,3 \%$ & $/$ & $100 \%$ \\
\hline Pas en emploi en 2003 & $/$ & $14,5 \%$ & $13,1 \%$ & $9,0 \%$ & $63,7 \%$ & $100 \%$ \\
\hline Ensemble & $40,5 \%$ & $23,7 \%$ & $16,3 \%$ & $8,3 \%$ & $11,2 \%$ & $100 \%$ \\
\hline
\end{tabular}

Augmentations du salaire: trajectoire où les changements de poste avec hausse de salaire sont plus nombreux que ceux avec baisse et vice versa pour les «diminutions du salaire».

Lire ainsi : 68,8\% des salariés soumis aux quatre contraintes de rythmes mesurées en 1998 et qui y sont encore soumis en 2003 n'ont pas connu de mobilité entre ces deux dates.

Champ: salariés de 15 à 59 ans soumis aux quatre contraintes de rythme en 1998; déclarations rétrospectives.

Source: enquête FQP (2003).

tion n'explique nullement que subir une contrainte de rythme liée aux contrôles de la hiérarchie favorise presque autant la mobilité ascendante que la mobilité descendante ( $c f$. tableau 9(5)). Ce constat concorde bien au contraire avec ce que nous savons des essais d'une forte intensité du travail. La pression hiérarchique ne s'exercerait pas seulement sur les «mauvais» travailleurs, mais sur tous, avec seulement des effets différents.

Reste toutefois une hypothèse que les données disponibles ne permettent pas de tester: la surveillance constante exercée par la hiérarchie permettrait à celleci de mieux connaître les performances des individus, favorisant ainsi la promotion des «bons» et l'éviction des «mauvais»; au contraire, en l'absence d'évalua-

(5) Estimations effectuées à la fois dans un modèle logit multinomial et selon la méthode du score de propension ( $c f$. infra). Les résultats étant très similaires, seuls ceux relatifs à la méthode du score de propension ont été reproduits dans le tableau 12. tion précise, les organisations où la hiérarchie joue un moindre rôle auraient une gestion plus rigide de leurs ressources humaines.

\section{Fuir le travail intense?}

Cela étant, la divergence des trajectoires marquées par une forte intensité du travail interroge la capacité qu'ont les salariés à soutenir une telle intensité: comment évolue l'intensité du travail avec la carrière? Les promotions et augmentations de salaire sont-elles associées à un maintien à un niveau élevé, voire à un renforcement, de l'intensité du travail? À l'inverse, les déclassements et diminutions de salaire s'accompagnent-elles nécessairement d'une diminution de l'intensité?

Pour les salariés connaissant un cumul maximal des contraintes de rythme en 1998, la diminution de l'intensité au cours des cinq années suivantes est fréquente. Elle concerne $38 \%$ d'entre eux, contre $44 \%$ qui restent au même niveau d'intensité et $18 \%$ qui ne sont plus en emploi en 2003. Dans les trois 


\section{Tableau 11 \\ Évolution de l'intensité et évolution salariale pour les salariés soumis à deux ou trois (sur quatre) contraintes de rythme en 1998}

\begin{tabular}{|c|c|c|c|c|c|c|}
\hline \multirow{2}{*}{$\begin{array}{l}\text { Évolution de l'intensité } \\
\text { entre } 1998 \text { et } 2003\end{array}$} & \multirow{2}{*}{ Pas de mobilité } & \multicolumn{3}{|c|}{ Évolution du salaire } & \multirow{2}{*}{$\begin{array}{l}\text { Mobilité sans } \\
\text { retour en } \\
\text { emploi }\end{array}$} & \multirow{2}{*}{ Ensemble } \\
\hline & & Augmentation & Stabilité & Diminution & & \\
\hline Augmentation & $32,3 \%$ & $35,2 \%$ & $23,5 \%$ & $9,1 \%$ & / & $100 \%$ \\
\hline Stabilité & $61,8 \%$ & $19,7 \%$ & $14,1 \%$ & $4,4 \%$ & 1 & $100 \%$ \\
\hline Diminution & $25,0 \%$ & $37,3 \%$ & $25,3 \%$ & $12,4 \%$ & I & $100 \%$ \\
\hline Pas en emploi en 2003 & / & $12,9 \%$ & $13,1 \%$ & $6,1 \%$ & $67,9 \%$ & $100 \%$ \\
\hline Ensemble & $41,1 \%$ & $23,5 \%$ & $17,1 \%$ & $6,8 \%$ & $16,8 \%$ & $100 \%$ \\
\hline
\end{tabular}

Augmentations du salaire: trajectoire où les changements de poste avec hausse de salaire sont plus nombreux que ceux avec baisse et vice versa pour les «diminutions du salaire».

Lire ainsi : 32,3\% des salariés soumis à deux ou trois des quatre contraintes de rythmes mesurées en 1998 et qui sont soumis en 2003 à un nombre de contraintes plus grand qu'en 1998, n'ont pas connu de mobilité entre ces deux dates.

Champ: salariés de 15 à 59 ans soumis à deux ou trois (sur quatre) contraintes de rythme en 1998; déclarations rétrospectives.

Source: enquête FQP (2003).

quarts des cas, la diminution s'effectue à l'occasion d'un changement de poste, et le plus souvent dans de bonnes conditions, c'est-à-dire sans perte de salaire ( $c f$. tableau 10). Certes, les salariés dont les contraintes de rythme diminuent connaissent un peu plus souvent que les autres une baisse de salaire. Mais globalement, les diminutions de salaire restent bien moins fréquentes que les situations d'augmentation ou de stabilité salariale. À court-moyen terme, une forte intensité du travail apparaît ainsi à la fois peu soutenable en termes de travail et relativement soutenable en termes de carrière.

Pour les salariés qui sont soumis à des contraintes de rythme moins nombreuses, l'évolution de l'intensité est un peu moins fréquente: elle concerne $29 \%$ des salariés, contre $54 \%$ qui restent au même niveau d'intensité et $17 \%$ qui ne sont plus en emploi en 2003. Pour ces salariés aussi, l'intensité a beaucoup plus tendance à diminuer qu'à augmenter et ces évolutions sont fortement liées à des changements de poste. En revanche, contrairement aux salariés soumis au cumul maximal des contraintes de rythme, la diminution de l'intensité n'est pas davantage liée à une diminution qu'à une augmentation de salaire. Les pertes de salaire sont même très rares (du moins sur cinq ans, et avec nos conventions de calcul; $c f$. tableau 11). Il est vraisemblable que l'intensité du travail est liée à de la mobilité parce que l'intensité n'est pas supportable à long terme. Mais cette mobilité ne prend pas toujours la forme d'une fuite avec baisse de l'intensité du travail au prix d'un sacrifice salarial, ce qui n'est d'ailleurs pas très surprenant du fait de possibilités souvent limitées de retour en emploi et d'une information partielle concernant le degré d'intensité des emplois potentiels.

Sur cette question, l'évolution avec l'âge de l'effet de l'intensité sur les trajectoires professionnelles apporte un éclairage complémentaire (cf. graphique 1). L'intensité est particulièrement difficile à supporter par les travailleurs âgés : ceux- ci ont en effet besoin d'utiliser des façons de faire particulières, tenant compte de la baisse de certaines capacités avec l'âge et une intensité trop élevée du travail ne le permet pas (PueYo, 1999). On peut donc s'attendre à ce que les liens de l'intensité du travail avec les formes descendantes de mobilité soient particulièrement nets pour les travailleurs âgés. De fait, en début de carrière, l'intensité du travail favorise les mobilités horizontales ou ascendantes; puis, progressivement, elle pèse sur les carrières salariales (la part des diminutions de salaire croît): pour les plus âgés enfin, elle pousse vers l'inactivité. Supposons un salarié qui est au début de sa carrière et demeure ensuite soumis à une forte intensité du travail. Supposons aussi que le lien entre intensité et mobilité demeure au fil du temps celui observé entre 1998 et 2003. La probabilité qu'il soit victime d'un «accident de carrière» socialement et psychologiquement douloureux serait assez forte.

Ainsi, de fortes contraintes de rythmes apparaissent à la fois peu soutenables à court terme du point de vue du travail et, également, peu soutenables à plus long terme cette fois du point de vue des carrières. En fait, tant qu'il existe des postes où le niveau d'intensité reste modéré, ou quand les salariés peuvent assez librement se partager le travail, les travailleurs âgés bénéficient d'une relative protection (PAILHÉ, 2006). Mais le travail le plus intense tend à retomber sur les jeunes (Millanvoye, Pueyo, 2006), ce qui induit que leurs trajectoires reproduisent celles des générations antérieures. Un tel partage trouve ses limites quand le vieillissement des salariés d'une entreprise fait qu'il n'y a plus assez de jeunes pour occuper tous les postes à forte charge. Compte tenu $\mathrm{du}$ vieillissement à venir de la population active française, une telle issue risque bien de concerner l'économie globale. In fine, l'intensification rendrait le travail peu soutenable pour la société tout entière dans la mesure où celle-ci vieillit et où la maind'œuvre jeune devient une ressource rare. 


\section{Discussion et perspectives}

Les résultats statistiques que nous venons d'exposer appuient donc l'idée d'un lien entre intensité du travail, mobilité accrue et divergence des carrières. Il convient néanmoins de réfléchir aux limites de leur valeur probante et aux prolongements souhaitables de notre travail.

\section{Les déclarations rétrospectives: une source de biais?}

L'utilisation de données rétrospectives pose des problèmes de fiabilité qui ont été largement étudiés et débattus (ThÉlot, 1990; Dex, 1995; Auriat, 1996). Dans le cas particulier de la mesure des conditions de travail, des travaux très précis ont examiné l'écart entre les déclarations des individus à une date donnée et le regard rétrospectif qu'ils portent ultérieurement sur cette même situation (MolinIÉ, 2003). On a pu ainsi montrer que les discordances, assez nombreuses, n'étaient pas le fait du hasard, mais avaient un sens. Pour intéressante que soit cette conclusion, elle est a priori plutôt gênante pour nous car il y a là une source potentielle non seulement de flou, mais encore de biais. En fait un examen détaillé des résultats obtenus par Anne-Françoise Molinié amène à la fois à reconnaître l'existence probable de biais et à penser que leur ampleur est limitée.

Les discordances entre les réponses formulées sur le moment et les réponses formulées rétrospectivement cinq ans plus tard sont d'autant plus grandes qu'elles portent sur des conditions de travail peu objectivées. Par exemple, le niveau de discordance est moyen pour un «travail répétitif sous contrainte de temps (à la chaîne, produit ou pièce qui se déplace, machine à cadence automatique, rythme imposé par une norme stricte) ». Cette question est pourtant particulièrement mal rédigée: mélange de deux registres différents (répétitivité et contrainte de rythme); liste longue difficile à mémoriser; usage d'un vocabulaire qui n'est pas celui des enquêtés («sous contrainte de temps»); «norme stricte» sans horizon temporel. De plus, la notion de travail répétitif est particulièrement floue, surtout quand on ne précise pas la durée du cycle. On peut donc en conclure que des questions précises sur les déterminants du rythme entraîneraient des taux de discordance plus faibles.

Molinié montre aussi qu'une des raisons des discordances est que l'enquêteur n'est pas le même, la sensibilité des réponses à la personnalité des enquêteurs étant largement admise (Gollac, 1997). Ceci ne joue pas dans notre cas, puisque les déclarations sont effectuées lors d'un seul entretien, par la même personne et auprès du même enquêteur.

De plus, Molinié montre qu'il n'y a pas de lien entre discordances des déclarations et évolution de l'état de santé. Bien qu'il n'y ait pas identité entre état de santé et situation professionnelle, ce résultat est important en ce qui nous concerne car l'impact de l'intensité du travail sur la santé est une des façons dont elle influe sur les carrières. Une source de biais potentielle se trouve ainsi relativisée.

Une autre source importante de discordances entre déclarations instantanées et rétrospectives est le fait qu'on s'accoutume à une situation et qu'on peut ne plus en percevoir les contraintes (y compris rétrospectivement). Ceci pourrait créer une liaison positive entre l'absence de changement de poste de 1998 à 2003 et l'absence de contraintes déclarées en 1998. Cependant on peut se demander ce qui advient à un individu qui intériorise les contraintes de rythme au point de ne plus en être conscient: peut-il néanmoins les respecter? De plus, si ce dernier biais était important, les contraintes de rythme déclarées rétrospectivement en 1998, dans le cadre de l'enquête FQP 2003, devraient être systématiquement plus faibles que celles déclarées sur le moment en 1998, dans le cadre de l'enquête sur les conditions de travail. Or, il n'en est rien. En effet, les déclarations rétrospectives et instantanées donnent des chiffres remarquablement concordants pour la plupart des indicateurs de conditions de travail ( $c f$. tableau 12), et notamment en ce qui concerne les contraintes de type « industriel» (cadence des machines, normes à respecter en un jour ou plus). En ce qui concerne la dépendance à la demande, le chiffre de FQP est plus faible, mais la formulation est différente, FQP parlant de la «demande du public», ce qui est restrictif. Inversement, les contrôles de la hiérarchie sont plus souvent cités dans FQP, donc en rétrospectif, peut-être en partie parce que le libellé de la question y est «contrôles permanents (ou quotidiens) », tandis que dans l'enquête sur les conditions de travail, la typographie semble permettre à l'enquêteur d'omettre l'extension "(ou quotidiens)».

Enfin, un dernier biais lié au questionnement rétrospectif sur les conditions de travail pourrait affecter nos résultats: les mobilités connues pendant cinq ans pourraient amener certains salariés à modifier les déclarations qu'ils font a posteriori de leurs contraintes de rythme et, ainsi, à surestimer le lien qui existe entre intensité du travail et mobilité. Mais, si tel est le cas, c'est que les salariés relient d'eux-mêmes (au moins indirectement) leur évolution professionnelle et l'intensité de leur travail. Et, sauf à penser que les gens se méprennent complètement sur leur situation, on peut considérer que le travail de reconstruction qu'effectue la mémoire correspond à des effets «objectifs », éventuellement amplifiés, mais bien réels.

\section{Causalité des phénomènes, problèmes d'endogénéité et de spécification}

Au-delà de la prise en compte de l'effet mémoire, nos réflexions méthodologiques ont été guidées par la volonté de nous approcher au plus près d'un 
effet «causal» de l'intensité sur la mobilité. Elles nous ont conduit à retenir un indicateur d'intensité qui s'appuie sur les contraintes de rythme et non sur l'intensité perçue, que la question «Êtes-vous obligé de vous dépêcher?» permet par exemple de mesurer. Par rapport à l'intensité perçue, le cumul des contraintes de rythme a comme avantage de se distinguer clairement de l'effort volontaire. Plus objectif, il a également pour avantage de ne pas dépendre a priori de l'état de santé et de limiter le risque de reconstruction du passé évoqué dans la section précédente.

Cependant, si cet indicateur permet d'éviter certains écueils, il ne nous prémunit pas de tout biais. Par exemple, les contraintes de rythme pourraient être liées à des caractéristiques non observées, telles que la politique de GRH de l'entreprise, qui influencent aussi la mobilité des salariés. Nous avons tenté de limiter l'ampleur de ce biais potentiel à partir des informations assez riches qui existent dans l'enquête FQP: les analyses «toutes choses égales par ailleurs» ont donc comme variables de contrôle le secteur et la catégorie socioprofessionnelle à un niveau détaillé, le fait d'être à temps partiel et, surtout, l'ancienneté dans le poste en 1998 et la trajectoire salariale à l'entrée dans ce poste.

L'effet de l'intensité sur la mobilité peut également être surestimé si certains salariés acceptent davantage de contraintes pour augmenter leurs chances de promotion. Mais, si les individus ne sont pas totalement irrationnels ou s'ils ne sont pas trompés par leur employeur, ceci ne se produit que s'il y a un lien «objectif» entre contraintes de rythme et promotion. On peut donc considérer que, si le biais existe, il est là encore d'ampleur limitée.

Par ailleurs, la spécification des logits ou des modèles de durée peut être critiquée. Lorsque, par exemple, on suppose que la probabilité de mobilité entre 1998 et 2003 pour un individu $\omega$ est donnée par:

$$
\operatorname{logit}(\operatorname{Pmob}(\omega))=\Sigma \mathrm{a}_{\mathrm{i}} \mathrm{X}_{\mathrm{i}}(\omega)+\mathrm{bI}(\omega),
$$

les $\mathrm{X}_{\mathrm{i}}$ étant des caractéristiques pertinentes des individus et I l'indicateur d'intensité (par exemple le nombre de contraintes de rythme), on suppose implicitement que l'effet de l'intensité du travail évalué à travers l'estimation du coefficient $b$, est le même pour tous les individus. Or, précisément, ce que nous savons de l'intensité du travail nous incite à penser qu'il n'en est rien. La méthode du score de propension (RUBIN, 1974; CRÉPON, IUNG, 1999; Askenazy, Carol, Marcus, 2002), permet, sous certaines conditions, de s'affranchir de cette hypothèse, probablement fort éloignée de la réalité, d'uniformité de l'effet de l'intensité.
Les investigations complémentaires auxquelles nous avons procédé(6) ( $c f$. tableau 12 pour un exemple) ne montrent pas de différence importante entre les résultats obtenus par la méthode du score de propension et ceux issus des logits.

\section{Tableau 12}

Comparaison des indicateurs de conditions de travail actuels et rétrospectifs, respectivement dans les enquêtes Conditions de travail 1998 et FQP 2003

\begin{tabular}{|l|c|c|}
\hline & \multicolumn{2}{|c|}{$\begin{array}{c}\text { Part de salariés dans } \\
\text { l'enquête... }\end{array}$} \\
\hline $\begin{array}{c}\text { Conditions } \\
\text { de travail } \\
1998\end{array}$ & FQP 2003 \\
\hline $\begin{array}{l}\text { Avoir un ou plusieurs salariés sous } \\
\text { ses ordres ou son autorité }\end{array}$ & $22,4 \%$ & $24,5 \%$ \\
\hline $\begin{array}{l}\text { Utiliser même occasionnellement } \\
\text { un micro-ordinateur, en réseau ou } \\
\text { non }\end{array}$ & $48,7 \%$ & $47,2 \%$ \\
\hline $\begin{array}{l}\text { Avoir son rythme de travail imposé } \\
\text { par: }\end{array}$ & $9,0 \%$ & $9,1 \%$ \\
\hline $\begin{array}{l}\text { La cadence automatique d'une } \\
\text { machine ou le déplacement auto- } \\
\text { matique d'un produit }\end{array}$ & $43,1 \%$ & $40,4 \%$ \\
\hline $\begin{array}{l}\text { Des normes de production, ou des } \\
\text { délais, à respecter en un jour au } \\
\text { plus }\end{array}$ & $64,5 \%$ & $46,2 \%$ \\
\hline $\begin{array}{l}\text { Une demande extérieure du public } \\
\text { obligeant ou non à une réponse } \\
\text { immédiate }\end{array}$ & $62,3 \%$ & $59,6 \%$ \\
\hline $\begin{array}{l}\text { Les contrôles ou surveillances } \\
\text { permanents (ou au moins quoti- } \\
\text { diens) exercés par la hiérarchie }\end{array}$ & $43,4 \%$ \\
\hline Être en contact direct avec le public & $6,4 \%$ & \\
\hline
\end{tabular}

Lorsqu'elles sont différentes de celles de l'enquête FQP, les formulations de l'enquête Conditions de travail sont indiquées en italique.

Lire ainsi: le pourcentage de salariés ayant en 1998 un ou plusieurs autres salariés sous leur autorité est de $22,4 \%$ si on se fie aux déclarations faites lors de l'enquête sur les conditions de travail réalisées cette année-là et de 24,5\% selon les déclarations rétrospectives faites lors de l'enquête FQP de 2003.

Champ: salariés de 15 à 59 ans en 1998; déclarations actuelles et rétrospectives.

Sources: enquêtes Conditions de travail de 1998 et FQP de 2003.

\section{Prolongements envisagés}

Qu'ils renvoient à la fiabilité des données rétrospectives utilisées ou aux problèmes d'endogénéité qui se posent dans l'interprétation causale des résultats, les différents biais présentés ne nous semblent à même d'invalider nos conclusions. Les résultats de nos analyses empiriques apparaissent ainsi robustes tant d'un point de vue interne qu'externe. Ils sont cohérents avec notre hypothèse initiale, elle-même formulée à partir d'une synthèse de travaux ergonomiques et sociologiques.

(6) Nous tenons à remercier Cédric Afsa et Bruno Crépon pour leurs suggestions sur ce point. Deux programmes implémentés, l'un par Emmanuel Duguet et l'autre par Cédric Afsa, ont été utilisés pour les estimations. Là encore, les résultats se sont révélés convergents. 
Pour aller plus loin et tenter de mettre en évidence statistiquement une causalité plus «pure», il faudrait des variables instrumentales qui permettent d'expliquer spécifiquement l'intensité du travail (à l'exclusion de la mobilité) ou des données de panel, ce qui n'est pas envisageable à court terme. Pour l'instant seule la deuxième vague de l'enquête longitudinale Santé et itinéraire professionnel (SIP), qui est prévue d'ici sept à huit ans, semble susceptible de fournir de telles données.

En attendant, d'autres analyses peuvent cependant être envisagées. Afin de préciser nos résultats, des modèles de durée à risques concurrents peuvent être estimés. D'autres méthodes d'analyse peuvent également être développées pour mieux contrôler de l'endogénéité des mécanismes analysés. Toujours dans un registre méthodologique, on peut procéder, à partir de données appariées entreprise-salariés, à une estimation du lien qui existe au niveau des établissements entre contraintes de rythme déclarées par les salariés et mouvements de main-d'œuvre mis en place par l'entreprise.

On souhaite par ailleurs analyser plus spécifiquement le lien entre intensité du travail et perte d'emploi (exclusion du marché du travail). Et on envisage de préciser les déterminants (caractéristiques de l'entreprise, du poste ou du salarié) des liens mis en évidence entre intensité et mobilité, d'une part, et entre mobilité et évolution de l'intensité, d'autre part. En particulier, quels rôles respectifs jouent l'origine sociale et le diplôme concernant l'effet de l'intensité sur la mobilité ? En d'autres termes, dans quelle mesure l'intensité contribue à reproduire des inégalités antérieures au travail lui-même?

Enfin, dans la perspective d'une meilleure compréhension du travail soutenable, nos résultats soulignent la nécessité d'analyser les effets de l'intensité du travail sur une durée plus longue que cinq ans, ce que suggèrent par ailleurs les observations de terrain et ce que devrait permettre la future enquête SIP.
À partir des données de l'enquête FQP, nous avons montré que l'intensité du travail était liée à la mobilité. L'hypothèse que nous avions formulée sur la base de la littérature était que l'intensité exerce des effets négatifs, mais aussi positifs sur les carrières et contribue à la divergence des trajectoires. Cette hypothèse n'est pas invalidée par nos résultats: lorsque les contraintes de rythme sont nombreuses, on observe à la fois davantage de promotions et d'augmentations de salaire et davantage de déclassements et de pertes de salaire.

Les trajectoires ascendantes étant bien plus fréquentes que les trajectoires descendantes, l'intensité peut finalement sembler jouer un rôle globalement positif. Pour autant, si elle est soutenable à court ou moyen terme du point de vue de la carrière, l'intensité du travail apparaît moins soutenable à plus long terme et, du point de vue du travail, même à court terme.

Afin de bien comprendre ces résultats, il faut se rappeler que les contraintes de rythme ne constituent pas une mesure de l'effort au travail, mais une mesure de la pression de l'organisation du travail. Nos analyses ne se limitent donc pas à la mise en évidence d'un mécanisme de sélection des salariés ayant le goût de l'effort, mais invitent à mieux comprendre l'effet des contraintes de rythme de travail sur la trajectoire des salariés.

L'intensité du travail paraît donc bien favoriser les trajectoires ascendantes et les trajectoires descendantes. Ceci ne signifie pas qu'elle n'ait, en moyenne, pas d'effet sur le rapport au travail. Subjectivement, les mobilités descendantes (plus rares) sont beaucoup plus marquantes que les mobilités ascendantes. Selon les déclarations des dirigeants d'entreprise, l'effet de démotivation associé est sans commune mesure avec les incitations salariales (BEwley, 1999). Par conséquent, via ses probables effets sur la mobilité, l'intensité excessive du travail risque d'avoir un effet négatif sur le bien-être et la motivation des salariés. Nos résultats apparaissent donc finalement assez cohérents avec ceux de BAUDELOT et al. (2002), qui indiquent que l'intensité est associée à (un peu) moins de bonheur au travail et beaucoup plus d'incertitudes.

Ils suggèrent que les réflexions sur la pénibilité du travail et sur la flexicurité doivent prendre en compte l'intensité du travail et qu'il demeure utile d'approfondir les recherches, qualitatives et quantitatives, pour préciser les conditions dans lesquelles une forte intensité du travail est plus ou moins susceptible de fragiliser les carrières. 


\section{Bibliographie}

Askenazy P., Caroli E., Marcus V., (2002), « New organizational practices and working conditions: evidence from France in the 1990's », Recherches économiques de Louvain, $\mathrm{n}^{\mathrm{o}}$ 1-2.

Amossé T., Chardon O., (2006), «Les travailleurs non qualifiés: une nouvelle classe sociale?», Économie et Statistique, no 393-394.

Amossé T., Gollac M., (2007), «Intensité du travail et trajectoire professionnelle : le travail intense est-il soutenable?», Document de travail du CEE, $\mathrm{n}^{\circ} 93$.

Auriat N., (1996), «Les défaillances de la mémoire humaine», Cahier, no 136, PUF - Ined.

Baudelot C., Gollac M., Bessière C., Coutant I., Godechot O., Serre D., Viguier F., (2002), Travailler pour être heureux?, Fayard.

Bewley T., (1999), Why wages don't fall during a recession, Harvard University Press.

Bosma H., Peter R., Siegrist J., Marmot M., (1998), «Two alternative job stress models and the risk of coronary heart disease », American Journal of Public Health, $\mathrm{n}^{\mathrm{o}} 171(5-6)$.

Bué J., Coutrot T., Hamon-Cholet S., Vinck L., (2007), «Conditions de travail: une pause dans l'intensification du travail», Premières Synthèses, no 01.2, Dares.

Cartron D., (2004), «L'intensification du travail», in Bué J., Coutrot T., Puech I. (coord.), Conditions de travail: les enseignements de vingt ans d'enquêtes, Octarès.

Chardon O., (2002), «La qualification des employés», Document de travail de l'Insee, $\mathrm{n}^{\circ} \mathrm{F} 0202$.

CréPON B., Iung N., (1999), «Innovations emploi et performance», Document de travail de la Direction des études et synthèses économiques, $\mathrm{n}^{\circ}$ 9904, Insee.

Desrosières A., ThÉvenot L., (1988), Les catégories socioprofessionnelles, La Découverte.

Dex S., (1995), «The reliability of recall data: a litterature review», Bulletin de méthodologie statistique, $\mathrm{n}^{\circ} 49$.

Docherty P., Forslin J., Shani A.B., (2002), Creating Sustainable Work Systems: Emerging Perspectives and Practice, Routledge.

Gollac M., (1997), «Des chiffres insensés», Revue Française de Sociologie.

Gollac M., (2005), «L'intensification du travail : formes et effets », Revue économique, $\mathrm{n}^{\circ} 2$.

Gollac M., Volkoff S., (1996), «Citius, altius, fortius, l'intensification du travail», Actes de la recherche en sciences sociales, 1996.

Gollac M., Volkoff S., (2001), «Intensité et fragilité», in Jeannot G. et Veltz P. (coord.), Le travail entre l'entreprise et la cité, Éditions de l'Aube.
InSEE (2005), «Enquête sur la formation et la qualification professionnelle 2003 », Insee Résultats et Sociétés, $n^{\circ} 48$.

KARASEK R., (1984), «Characteristics of Task Structure Associated with Physiological Stress and CardioVascular Diseases ", Annals of the American Conference of Governmental Industrial Hygienists, vol. 8.

Karasek R., Theorell T., (1990), Healthy Work: stress, productivity, and the reconstruction of the working life, Basic Books.

Laville A., (1998), L'ergonomie, PUF, 6 ${ }^{\mathrm{e}}$ édition corrigée.

LePlat J., Cuny X., (1984), Introduction à la psychologie du travail, PUF.

Millanvoye M., Pueyo P., (2006), «Organisation du travail, vieillissement des salariés et intensification du travail», in Askenazy Ph., Cartron D., de Coninck F., Gollac M. (coord.), Organisation et intensité du travail, Octarès, Toulouse.

Molinié A.-F., (2003), «Interroger les salariés sur leur passé professionnel: le sens des discordances», Revue d'Épidémiologie et de Santé publique, $\mathrm{n}^{\mathrm{O}} 51$.

Niedhammer I., Teck M.L., Starke D., Siegrist J., (2004), «Effort-Reward Imbalance Model and self reported health: Cross-sectional and prospective results from the GAZEL Cohort», Social Science \& Medicine, vol. 58, $\mathrm{n}^{\circ} 8$.

PAilhÉ A., (2006), «L'intensité du travail s’allège-t-elle avec l'âge?», in Askenazy Ph., Cartron D., de Coninck F., Gollac M. (coord.), Organisation et intensité $d u$ travail, Octarès, Toulouse.

Paugam S., (2000), Le salarié de la précarité. Les nouvelles formes de l'intégration professionnelle, PUF.

Pueyo V., (1999), Régulation de l'efficience en fonction de l'âge et de l'expérience professionnelle, thèse de doctorat d'ergonomie, EPHE.

RuBin D., (1974), «Estimating causal effect of treatments in randomized and nonrandomized studies », Journal of Educational Psychology, ${ }^{\circ} 66$.

SiEgRIST J., (1996), «Adverse health effects of high effort - low reward conditions at work», Journal of Occupational Health Psychology, no 1, 27-43.

ThÉlot C., (1990), «L'erreur de mémoire sur la situation professionnelle», in Les ménages: mélanges en l'honneur de J. Desabie, Insee.

VAlEyre A., (2004), «Forms of work intensification and economic performance in French manufacturing», Eastern Economic Journal, vol. 30, no 4.

WICHERT I., (2002), «Job insecurity and job intensification: the effects on health and well-being», in Burchell B., Ladipo D., Wilkinson F. (dir.), Job Insecurity and Work Intensification, Routledge. 